\title{
Via de Parto e Resultados Perinatais em Gestantes Diabéticas
}

\author{
Route of Delivery and Perinatal Outcomes of Diabetic Pregnant Women
}

Belmiro Gonçalves Pereira, Aníbal Faúndes, Mary Angela Parpinelli, Renato Passini Jr. Eliana Amaral, Helaine Besteti Pires, José Guilherme Cecatti

\section{RESUMO}

Objetivo: apresentar os resultados perinatais obtidos a partir da aplicação de um protocolo de assistência às gestantes diabéticas no Centro de Atenção Integral à Saúde da Mulher (CAISM) da UNICAMP.

Métodos: foram estudadas 90 gestantes diabéticas que iniciaram controle pré-natal na instituição e foram submetidas a este protocolo. Foram comparadas com dois grupos controles de 180 gestantes cada: um constituido por gestantes pareadas por idade e número de gestações (controle A) e outro por gestantes aleatoriamente selecionadas (controle B). Nos três grupos foram avaliadas as seguintes variáveis: tipo de parto, indicações de cesárea, idade gestacional, índice de Apgar ao primeiro e quinto minuto de vida, peso e adequação de peso para idade gestacional, morbidade e mortalidade perinatal. Para a análise estatistica utilizaram-se médias, desvio-padrão, os testes t de Student e do $\chi^{2}$.

Resultados: entre as gestantes diabéticas ocorreu maior incidência de cesáreas, recémnascidos prematuros e grandes para a idade gestacional (GIG), assim como uma maior freqüencia de patologias neonatais (hipoglicemia, hipocalcemia, hiperbilirrubinemia, desconforto respiratório e depressão neonatal). A incidência de Apgar $<7$ e a mortalidade perinatal foram significativamente maiores do que no grupo controle aleatoriamente selecionado, mas a diferença desapareceu quando se comparou ao grupo controle pareado por idade e número de gestações.

Conclusões: apesar de o protocolo visar um perfeito controle metabólico nas gestantes diabéticas, os resultados perinatais ainda são desfavoráveis em comparação às gestantes não-diabéticas.

PALAVRAS-CHAVE: Mortalidade perinatal. Diabetes mellitus. Resolução da gestação.

\section{Introdução}

O diabete é uma doença metabólica caracterizada por hiperglicemia que, posteriormente, pode causar alterações vasculares, renais, cardíacas e oculares. A alteração do metabolismo é também responsável pelas principais alterações na evolução dos fetos e recémnascidos das gestantes com esta doença ${ }^{1,2}$.

Tradicionalmente, o impacto de um bom acompanhamento pré-natal é avaliado pelo parto de uma criança viva, sadia e que sobreviva ao

Disciplina de Obstetrícia, Departamento de Tocoginecologia, Faculdade de Ciências Médicas, Universidade Estadual de Campinas

Correspondência:

Belmiro Gonçalves Pereira

Rua Alexander Fleming, 101

Caixa Postal 6030

13081-970 - Campinas - São Paulo

Fax: (19) 788-9304 período neonatal. Em 1978, Kitzmiller et al. ${ }^{3}$ analisaram a ocorrência de resultados perinatais desfavoráveis em 147 gestantes diabéticas. Estes autores encontraram $7,6 \%$ de síndrome de desconforto respiratório, 9\% de anomalias congênitas, $22 \%$ de hipocalcemia, $19 \%$ de hiperbilirrubinemia, $47 \%$ de hipoglicemia e $36 \%$ de macrossomia, verificando que a taxa de sobrevida foi de $96,6 \%$.

Examinando a relação entre a normoglicemia materna e a evolução fetal e neonatal em gestantes diabéticas, conclui-se que, quando se consegue bom controle metabólico, a morbidade perinatal é comparável à de gestantes normais independente da idade, paridade e gravidade da doença ${ }^{4}$.

A freqüência de malformações e morte perinatal é, atualmente, menor e compatível com os resultados obtidos em grávidas não-diabéticas, após a introdução de terapia com insulina e de protocolos de assistência mais rígidos no 
seguimento destas gestantes ${ }^{5}$. Em 1984, Olofsson et al. $^{6}$ apresentaram indice de $2,4 \%$ de perdas neonatais causadas, em geral, pelas malformações. Ainda hoje, as incidências de malformações e de morte perinatal, apesar de baixas, são avaliadas, pois são indicadores da qualidade de assistência à gestante, variáveis na dependência do serviço, nível sócio-econômico e outros fatores ${ }^{7}$.

Já a morbidade perinatal pode refletir melhor a qualidade de assistência oferecida à mulher diabética. Dessa forma, avalia-se a ocorrência de hipoglicemia, hipocalcemia, hiperbilirrubinemia e síndrome de desconforto respiratório, entre outros ${ }^{4}$.

Partindo do princípio que a qualidade do atendimento oferecido à gestante diabética é medido, com maior precisão, pelos resultados perinatais, e que existe um protocolo de atendimento a estas mulheres, em uso no serviço há vários anos, visando o controle rígido da doença ${ }^{8}$, do ponto de vista de controle metabólico, dietético, educacional, da evolução da gestação e do desenvolvimento e vitalidade fetais, parece oportuno realizar esta avaliação.

\section{Pacientes e Métodos}

Este é um estudo prospectivo e descritivo da população de grávidas diabéticas submetidas a um protocolo de assistência no Pré-Natal Especializado (PNE) da Maternidade do Centro de Atenção Integral à Saúde da Mulher (CAISM) da Universidade Estadual de Campinas (UNICAMP), comparativamente a dois grupos distintos de gestantes atendidas no mesmo período. Os procedimentos clínicos e de avaliação materna e feto/neonatal adotados neste protocolo de seguimento das gestantes diabéticas incluem consultas periódicas e freqüentes ao pré-natal, internações periódicas para avaliações do estado metabólico e ajustes das doses de insulina, além da vitalidade fetal ${ }^{8}$.

Foram estudados os resultados perinatais obtidos dos prontuários de 90 gestantes diabéticas, comparados com os observados em dois grupos controles de 180 gestantes cada, um deles pareado por idade e número de gestações (controle A) e outro selecionado aleatoriamente (controle B).

As seguintes variáveis foram avaliadas: tipo de parto e indicação de cesárea, idade gestacional, Apgar ao primeiro e quinto minuto de vida peso e adequação de peso para a idade gestacional segundo curva de Lubchenco ${ }^{9}$, hipoglicemia, hipocalcemia, hiperbilirrubinemia, policitemia, distúrbios respiratórios, malformações e morte perinatal.

Para análise estatística, foram utilizados a média, o desvio-padrão, o teste $t$ de Student para amostras pareadas ou não, conforme o grupo controle (A ou B). Os testes $\chi^{2}$ e $\chi^{2}$ para tendência foram utilizados para as variáveis categóricas. O projeto foi previamente aprovado pela Comissão de Ética da instituição.

\section{Resultados}

Entre as diabéticas houve $72,2 \%$ de cesáreas e nos dois grupos controles houve $30 \%$, sendo estas diferenças estatisticamente significativas. No entanto, $46,2 \%$ das cesáreas no grupo de pacientes diabéticas foram indicadas por antecedente de cesárea e, portanto, realizadas no início do trabalho de parto ou eletivamente. A freqüência desta indicação foi maior no grupo de estudo do que no grupo controle $\mathrm{B}$, que teve número significativamente maior de indicações por apresentação pélvica que o grupo de estudo (Tabela 1).

Tabela 1 - Distribuição das gestantes diabéticas e controles de acordo com a via de parto e indicação de cesárea

\begin{tabular}{|c|c|c|c|c|c|c|}
\hline \multirow[b]{2}{*}{ Indicação de cesárea } & \multicolumn{2}{|c|}{ Diabéticas } & \multicolumn{2}{|c|}{ Controle A } & \multicolumn{2}{|c|}{ Controle B } \\
\hline & $\mathrm{n}$ & $\%$ & $\mathrm{n}$ & $\%$ & $\mathrm{n}$ & $\%$ \\
\hline De repetição & 30 & 46,2 & 20 & $37,0^{(*)}$ & 12 & $22,6^{(*)}$ \\
\hline Sofrimento fetal & 17 & 26,1 & 10 & $18,5^{(*)}$ & 13 & $24,5^{(*)}$ \\
\hline Hipertensão arterial & 8 & 12,3 & 2 & $3,7^{(*)}$ & 6 & $11,3^{(*)}$ \\
\hline Diabetes & 4 & 6,1 & - & - & - & - \\
\hline Acidente de cordão & 2 & 3,0 & - & - & - & - \\
\hline Apresentação pélvica & 2 & 3,0 & 4 & $7,4^{(*)}$ & 10 & $18,8^{(* *)}$ \\
\hline Gravidez prolongada & 1 & 1,5 & 1 & $3,7^{(*)}$ & 1 & $1,8^{(*)}$ \\
\hline DCP\# & 1 & 1,5 & 4 & 7,4 & 4 & 7,5 \\
\hline Outras & - & - & 12 & 22,2 & 7 & 13,2 \\
\hline Total de cesáreas & $65^{(+*+1)}$ & & 54 & & 53 & \\
\hline
\end{tabular}

$\#$ - DCP = Desproporção Céfalo-Pévica

(") n.s.

(") $p<0,01$

("') Cesárea $x$ vaginal $p<0,001$ 
Houve diferenças entre os grupos de estudo e controles, quanto à idade gestacional dos RN, principalmente pela maior porcentagem de resoluções antes da 36 a semana de gestação e menor porcentagem de RN com mais de 40 semanas entre as gestantes diabéticas. Entre as diabéticas, a porcentagem de partos antes da $36^{\mathrm{a}}$ semana foi aproximadamente o dobro da observada nos grupos controles (Tabela 2).

Tabela 2 - Distribuição das gestantes diabéticas e controles de acordo com a idade gestacional ao nascimento.

\begin{tabular}{|c|c|c|c|c|c|c|}
\hline \multirow[b]{2}{*}{ Idade gestacional (semanas) * } & \multicolumn{2}{|c|}{ Diabéticas } & \multicolumn{2}{|c|}{ Controle $A^{(\&)}$} & \multicolumn{2}{|c|}{ Controle B (2\&) } \\
\hline & $\mathrm{n}^{\star *}$ & $\%$ & $\mathrm{n}^{\star \star *}$ & $\%$ & $\mathrm{n}^{\star \star * *}$ & $\%$ \\
\hline Até 28 & 1 & 1,1 & 2 & 1,1 & 0 & - \\
\hline$>28-32$ & 2 & 2,2 & 2 & 2,2 & 1 & 0,6 \\
\hline$>32-36$ & 13 & 14,4 & 9 & 5,0 & 12 & 6,6 \\
\hline$>36-40$ & 56 & 62,2 & 90 & 50,0 & 90 & 50,0 \\
\hline$>40$ & 18 & 20,0 & 75 & 41,7 & 77 & 42,8 \\
\hline Total & 90 & 100 & 180 & 100 & 180 & 100 \\
\hline
\end{tabular}

(") Idade gestacional até $36 \times$ maior que 36 semanas $p<0,05$

(\&) Diabéticas $x$ controle $A: x^{2}=12,308 p<0,001$

(\&\&) Diabéticas $x$ controle $B: x^{{ }^{t}}=17,280 p<0.001$

** 4 natimortos

*** 3 natimortos

**** 2 natimortos

Quanto ao peso dos recém-nascidos, verificou-se que a média foi significativamente maior para os filhos de mães diabéticas $(3.459,9 \pm$ $738,8)$ em relação aos controles $(3.107,1 \pm 703,2$ e $3.089,9 \pm 610,7$, respectivamente para os grupos
A e B). Esta diferença deve-se, fundamentalmente, ao fato de $52 \%$ dos filhos de mães diabéticas terem tido peso superior a 3.500 g em comparação com $29 \%$ do controle A e $20 \%$ do controle aleatório (B) (Tabela 3).

Tabela 3 - Distribuição das gestantes diabéticas e controles de acordo com o peso dos recém-nascidos e adequação do peso para idade gestacional (IG) ${ }^{9}$.

\begin{tabular}{|c|c|c|c|c|c|c|}
\hline \multirow[b]{2}{*}{ Peso do RN (g) } & \multicolumn{2}{|c|}{ Diabéticas } & \multicolumn{2}{|c|}{ Controle A } & \multicolumn{2}{|c|}{ Controle B } \\
\hline & $\mathrm{n}$ & $\%$ & $n$ & $\%$ & $n$ & $\%$ \\
\hline Até 1.499 & 3 & 3,3 & 6 & 3,3 & 5 & 2,8 \\
\hline $1.500-2.499$ & 3 & 3,3 & 23 & 12,7 & 21 & 11,6 \\
\hline $2.500-3.499$ & 37 & 41,1 & 99 & 55,0 & 118 & 85,5 \\
\hline $3.500-3.999$ & 32 & 35,5 & 42 & 23,3 & 29 & 16,6 \\
\hline 4.000 ou mais & 15 & 16,6 & 10 & $5,5^{(*)}$ & 7 & $3,8^{(*)}$ \\
\hline \multicolumn{7}{|l|}{ Adequação à IG } \\
\hline Pequeno & 5 & 5,8 & 19 & 10,9 & 17 & 9,5 \\
\hline Adequado & 54 & 62,7 & 127 & 71,7 & 140 & 78,6 \\
\hline Grande & 27 & 31,3 & 31 & $17,5^{(*)}$ & 21 & $11,7^{(*)}$ \\
\hline Total & 90 & 100,0 & 180 & 100,0 & 180 & 100,0 \\
\hline
\end{tabular}

Quanto à posição na curva de Lubchenco ${ }^{9}$, foram observadas diferenças estatisticamente significativas entre os RN das pacientes diabéticas e os dos grupos controles. A porcentagem de recém- nascidos grandes para a idade gestacional (GIG) foi aproximadamente duas vezes maior no grupo de estudo do que no controle A e cerca de três vezes maior que no controle B (Tabela 3). 
O índice de Apgar ao primeiro minuto de vida também foi diferente nos três grupos, embora só houvesse diferença estatística entre o grupo de estudo e o controle B. Entre as diabéticas, a porcentagem de $\mathrm{RN}$ deprimidos foi de $16,2 \%$, praticamente o dobro do controle aleatório. Quanto ao Apgar do quinto minuto de vida, não houve diferenças entre os grupos (Tabela 4).

Tabela 4 - Distribuição das gestantes diabéticas e controles com baixo índice de Apgar ao primeiro e quinto minutos de vida.

\begin{tabular}{|c|c|c|c|c|c|c|}
\hline \multirow[b]{2}{*}{ Índice de Apgar < 7} & \multicolumn{2}{|c|}{ Diabéticas } & \multicolumn{2}{|c|}{ Controle A } & \multicolumn{2}{|c|}{ Controle B } \\
\hline & $\mathrm{n}$ & $\%$ & $\mathrm{n}$ & $\%$ & $\mathrm{n}$ & $\%$ \\
\hline $1^{\circ}$ minuto & 14 & 16,2 & 23 & $12,9^{(*)}$ & 14 & $7,8^{(*)}$ \\
\hline $5^{\circ}$ minuto & 0 & 0,0 & 7 & $3,9^{(*)}$ & 1 & $0,5^{(*)}$ \\
\hline Total & $86^{*}$ & 100,0 & $177^{*}$ & 100,0 & $178^{*}$ & 100,0 \\
\hline
\end{tabular}

(") n.s.

(") $p<0,05$

* Excluídos os natimortos

A distribuição da morbidade neonatal dos RN das diabéticas em relação aos controles apresentou diferenças significativas quanto à ocorrência de hiperbilirrubinemia, hipoglicemia e policitemia. A porcentagem de síndrome de desconforto respiratório foi mais elevada entre os filhos de diabéticas do que nos controles, porém só houve significância estatística quando comparado ao controle B. A hipocalcemia e as malformações congênitas tiveram distribuição igual nos três grupos (Tabela 5).

Tabela 5 - Distribuição das principais etiologias de morbidade neonatal dos recém-nascidos de mães diabéticas e controles.

\begin{tabular}{|c|c|c|c|c|c|c|}
\hline \multirow[b]{2}{*}{ Morbidade neonatal } & \multicolumn{2}{|c|}{ Diabéticas } & \multicolumn{2}{|c|}{ Controle A } & \multicolumn{2}{|c|}{ Controle B } \\
\hline & $\mathrm{n}$ & $\%$ & $\mathrm{n}$ & $\%$ & $n$ & $\%$ \\
\hline $\mathrm{Sem}^{*}$ & 46 & 51,1 & 145 & $80,5^{(4)}$ & 152 & $84,4^{(4)}$ \\
\hline Hiperbilirrubinemia & 23 & 26,1 & 15 & $8,4^{(4)}$ & 15 & $8,4^{(4)}$ \\
\hline Hipoglicemia & 17 & 19,7 & 0 & $0,0^{(4)}$ & 0 & $0,0^{(4)}$ \\
\hline Desconforto respiratório & 8 & 9,3 & 7 & $3,9^{(1)}$ & 4 & $2,2^{(3)}$ \\
\hline Policitemia & 5 & 5,8 & 0 & $0,0^{(2)}$ & 0 & $0,0^{(2)}$ \\
\hline Hipocalcemia & 1 & 1,1 & 0 & $0,0^{(1)}$ & 0 & $0,0^{(1)}$ \\
\hline Malformações & 3 & 3,4 & 2 & $1,1^{(1)}$ & 4 & $2,2^{(1)}$ \\
\hline Outras & 0 & & 8 & & 3 & \\
\hline Sem diagnóstico & 2 & & 3 & & 2 & \\
\hline
\end{tabular}

(1) n.s.

(2) $p<0,01$

(3) $p<0,05$

(4) $\mathrm{p}<0,001$
* sem patologia $x$ com patologia $p<0,001$

A mortalidade perinatal foi maior no grupo de diabéticas que nos controles, apesar de os números serem pequenos para identificar diferenças estatisticamente significativas com o controle pareado. Quando comparados com o controle aleatório, foram encontradas diferenças estatisticamente significativas. Houve 6 casos de mortes perinatais entre as diabéticas, sendo quatro delas natimortos (Tabela 6). Entre estas perdas perinatais foi conseguido o diagnóstico etiológico por necrópsia em três casos: um natimorto portador de deformidade cardíaca grave, um RN que morreu por cardiopatia e outro por septicemia. 
Tabela 6 - Morbidade perinatal entre os filhos de gestantes diabéticas e de controles.

\begin{tabular}{|c|c|c|c|c|c|c|}
\hline & \multicolumn{2}{|c|}{ Diabéticas } & \multicolumn{2}{|c|}{ Controle A } & \multicolumn{2}{|c|}{ Controle B } \\
\hline & $n$ & $\%$ & $n$ & $\%$ & $\mathrm{n}$ & $\%$ \\
\hline Natimorto & 4 & 4,4 & 3 & $\left.1,7^{*}\right)$ & 2 & $1,1^{(* *)}$ \\
\hline Morte neonatal & 2 & 2,2 & 3 & $1,7^{(*)}$ & 1 & $0,5^{(*)}$ \\
\hline Morte perinatal & 6 & 6,7 & 6 & $3,3^{(*)}$ & 3 & $1,7^{(* *)}$ \\
\hline Total & 90 & 100,0 & 180 & 100,0 & 180 & 100,0 \\
\hline
\end{tabular}

(*) n.s.

(") $p<0,05$

\section{Discussão}

Os resultados deste estudo mostram elevada freqüência de partos por cesárea, complicações neonatais e mortalidade entre os fetos e recémnascidos das gestantes com diabetes. Estes comparam-se favoravelmente às estatísticas internacionais, ainda que com a restrição de que os casos do presente estudo tenham sido de diabetes gestacional ou de evolução mais recente ${ }^{10}$.

Não se pode atribuir a maior incidência de cesárea entre as gestantes diabéticas à idade e à maior paridade, visto que a diferença mantém-se tanto para os controles pareados por estas variáveis, quanto para os controles aleatórios. É verdade que no grupo houve alta incidência de hipertensão, sugerindo que esta intercorrência possa justificar a maior incidência de cesárea. Entretanto, o grupo controle A também apresentou elevada porcentagem de hipertensão, três vezes maior que no controle B, e sua taxa de cesárea foi igual à observada neste grupo.

Os 15\% de casos de diabéticas com polidrâmnio poderiam explicar apenas parcialmente esta maior incidência de cesárea, pois a diferença nas taxas, de $30 \%$ para $70 \%$, é bem maior que estes $15 \%$. Outra parte da diferença pode ser explicada pela maior freqüência de cesárea anterior, 9\% maior que no grupo controle pareado por idade e número de gestações. Porém, este conjunto de possíveis explicações não chegam a convencer plenamente.

Há de se aceitar que, sendo a gestante diabética, já existe um ambiente de insegurança sobre o risco fetal e materno, levando à maior propensão para se interromper a gravidez ou trabalho de parto. Soma-se ainda a maior proporção destas pacientes que chegam ao parto com solicitação aprovada de ligadura tubária, seguindo a sistemática da instituição. Mesmo sendo norma do serviço não indicar cesárea para se fazer laqueadura, o obstetra, em caso de dúvida sobre a melhor via de parto, poderia sentir-se mais inclinado à cesárea, sobretudo quando sabe que será o último parto desta paciente e que a cicatriz não ofereceria riscos para futuras gestações.

Neste estudo, a incidência de cesárea aproximou-se da encontrada por Kitzmiller et al. ${ }^{3}$, que foi de $69 \%$, tendo o antecedente de cesárea representado $40 \%$ das indicações. Todavia, Olofsson et al. ${ }^{6}$ relataram apenas $45 \%$ de cesárea e a principal indicação foi sofrimento fetal, seguido de desproporção cefalopélvica e, só depois, o antecedente de cesárea. Os resultados de Olofsson, cuja casuística tinha predomínio de diabetes classe $\mathrm{C}$ ou mais grave, sugerem que, com indicações mais criteriosas, é possivel reduzir a incidência de cesáreas e, em parte, as iatrogenias dela decorrentes, tanto para o lado materno quanto aos resultados perinatais ${ }^{6}$.

Em relação ao peso dos RN, observou-se que nos filhos das diabéticas, este foi em média $360 \mathrm{~g}$ superior ao dos controles, e a incidência de recémnascidos com peso superior a $4.000 \mathrm{~g}$ foi três a cinco vezes maior do que a observada em quaisquer dos controles. O peso mais elevado dos RN de mães diabéticas foi semelhante àquele relatado por Oliveira et al. ${ }^{11}$. A incidência de $17 \%$ de RN com peso superior a $4.000 \mathrm{~g}$ foi maior do que a de $11,5 \%$ descrita por Kitzmiller et al. ${ }^{3}$. Por outro lado, em relação à idade gestacional por ocasião do parto, as pacientes diabéticas tiveram praticamente o dobro de prematuros, quando comparadas aos controles. No entanto, esta diferença limitou-se apenas aos prematuros com idade gestacional superior a 32 semanas.

Os dados deste estudo são muito semelhantes àqueles referidos na literatura. Kitzmiller et al. $^{3}$, por exemplo, referiram incidência de $20 \%$ de recém-nascidos com até 36 semanas. Olofsson et al. ${ }^{6}$ relatou incidência geral de 17,4\% de RN com menos de 37 semanas e Von Kries et al. ${ }^{12}$ mostraram $21 \%$ de prematuros.

É possivel que com melhores condições de assistência pré-natal às diabéticas seja alcançada redução dos índices de prematuridade, como demonstraram Olofsson et al. ${ }^{6}$ em três períodos 
distintos em que assistência pré-natal foi sendo melhorada de acordo com a avaliação dos resultados e aplicação de novos conhecimentos. Com isto, estes índices foram sendo reduzidos de $22,9 \%$ para $16,5 \%$ e, mais recentemente, para $14 \%$.

Com a média de peso mais elevada e idade gestacional mais baixa, não surpreende que a proporção de recém-nascidos GIG e pequenos para idade gestacional (PIG) seja diferente quando se compara o grupo das diabéticas com os controles. Os 31\% de RN considerados GIG no presente estudo são, no entanto, inferiores àqueles encontrados por Kitzmiller et al. ${ }^{3}$, de 36,1\%. Já a freqüência de recém-nascidos PIG nas diabéticas aqui analisadas foi de $5,8 \%$, superior aos $3,5 \%$ das pacientes daquele estudo ${ }^{3}$.

A maior freqüência de recém-nascidos GIG entre as diabéticas é indicador da dificuldade de se obter controle metabólico ideal durante a gestação. Foi isto que suscitou o desenvolvimento de uma proposta para melhorar o controle e, com isso, melhorar os resultados.

Apesar da maior incidência de prematuridade, que poderia interferir nos outros resultados perinatais, os índices de Apgar ao primeiro e ao quinto minuto de vida não foram diferentes entre o grupo de diabéticas e o controle com igual idade e número de gestações, mas a incidência de depressão neonatal foi significativamente mais freqüente entre as diabéticas do que entre as pacientes do controle aleatório. A incidência de $16 \%$ no grupo de estudo foi elevada, chegando a duas vezes a incidência observada na população considerada de baixo risco e podendo ser explicada tanto pela prematuridade, quanto pela maior incidência de cesárea. Contudo, os 16\% de Apgar menor que 7 deste estudo e a ausência de Apgar menor que 7 aos cinco minutos de vida, nos nascidos vivos de gestantes diabéticas, mostram o bom atendimento neonatal e ilustram as vantagens do trabalho em equipe.

A observação geral dos diferentes indicadores de resultados perinatais sugere que a maior diferença esteja no elevado índice de natimortalidade entre os filhos de diabéticas. Infelizmente, a presente casuística é ainda relativamente pequena para distinguir diferenças em fenômenos de baixa freqüência como este, embora tenha sido duas vezes mais freqüente que nos controles pareados e quatro vezes maior que nos controles aleatórios.

Outra diferença observada corresponde à morbidade neonatal. Apenas 46 dos 86 nascidos vivos (54\%) no grupo das diabéticas não tiveram complicações, comparados com mais de $80 \%$ dos controles. A incidência de $19,7 \%$ de hipoglicemia observada no grupo de diabéticas foi menor que a relatada por Kitzmiller et al. ${ }^{3}$, de $47 \%$. A incidência de hiperbilirrubinemia de $27 \%$ dos nativivos foi, também, significativamente superior àquela encontrada entre os controles e semelhante à relatada na literatura.

Outra complicação neonatal, referida na literatura como sendo mais freqüente entre os filhos de mães diabéticas, é o desconforto respiratório. Isto foi observado na população do grupo de estudo, que apresentou incidência desta complicação duas a quatro vezes superior à encontrada entre os $\mathrm{RN}$ dos grupos controles (A ou B). A incidência de 9,3\% neste estudo foi maior que a encontrada por Olofsson et al. ${ }^{6}$, de $16,8 \%$ e por Kitzmiller et al. ${ }^{3}$, de $14 \%$.

A incidência de malformações entre as pacientes diabéticas foi bastante baixa e semelhante à observada entre os controles, diferindo da literatura. Pedersen e MolstedPedersen ${ }^{13}$ e Olofsson et al. ${ }^{6}$ relataram incidência de malformações de $8 \%$ e $6,8 \%$ respectivamente. Vale, contudo, frisar que a maioria das diabéticas acompanhadas neste estudo foi classificada como gestacional e, conseqüentemente, menos predispostas a malformações, que são fenômenos de baixa prevalência entre as diabéticas menos graves $^{3,6,13}$.

A mortalidade perinatal de $6,6 \%$ observada nos filhos de mães diabéticas deste estudo ficou entre os 10,9\% encontrados por Olofsson et al. ${ }^{6} \mathrm{e}$ os $3,4 \%$ encontrados por Kitzmiller et al. ${ }^{3}$. Em séries mais recentes, a mortalidade perinatal ocorre entre $2,8 \%{ }^{12}$ e $5 \%$ nos casos mais graves da doença $^{14}$.

A despeito de controle pré-natal sucessivamente mais cuidadoso e de um melhor entendimento da interação fisiopatológica da diabete e da gestação, os resultados perinatais ainda não chegam a ser tão bons quanto os da mulher não-diabética. Isto estimula tomar medidas que permitam melhorar ainda mais o prognóstico perinatal destas pacientes.

\section{SUMMARY}

Purpose: to present the perinatal outcomes resulting from the use of a protocol for assistance to diabetic pregnant women used at the Center for Integral Assistance to Women's Health (CAISM), of the University of Campinas.

Methods: ninety diabetic pregnant women, who were assisted at the institution with this protocol, were compared with two control grups: the first consisted of 180 pregnant women with equal number of gestations and same age (control $A)$ and the second consisted of 180 randomly selected pregnant women 
(control B). The study variables were route of delivery, indication for cesarean section, gestational age, Apgar score at first and fifth minute, weight, adequacy of weight for gestational age and perinatal morbidity and mortality. For the statistical analysis Student's t-test and the $\chi_{2}$ test were used.

Results: there was a higher incidence of cesarean sections, prematures and large to gestational age (LGA) babies among diabetic women, as well as higher occurrence of neonatal morbidity such as hypoglycemia, hypocalcemia, hyperbilirubinemia, respiratory distress and neonatal depression. The incidence of low Apgar score and perinatal mortality was significantly higher than in the randomly selected group, but the same as in the group matched regarding age and number of pregnancies.

Conclusions: although this protocol intends to obtain a perfect metabolic control among diabetic pregnant women, the perinatal outcomes are still unfavorable in comparison to nondiabetic pregnant women.

KEY WORDS: Delivery. Perinatal outcomes. Pregnancy. Diabetes.

\section{Referências}

1. Borden G. Metabolismo energético na gravidez e no diabete melito gestacional. Clín Obstet Ginecol Am Norte. 1996; 1:1-12.

2. Honko CJ, Khandelwal M. Monitorização da glicose e terapia insulínica durante a gravidez. Clin Obstet Ginecol Am Norte. 1996; 1:49-76.

3. Kitzmiller JL, Cloherty JP, Younger MD, Tabatabaii A, Rothchild SBb, Sosenko I, et al. Diabetic pregnancy and perinatal morbidity. Am J Obstet Gynecol 1978; 131:560-80.
4. Tyrala EE. O bebê da mãe diabética. Clin Obstet Ginecol Am Norte. 1996; 1:217-52.

5. Reece EA, Eriksson UJ. Patogênese das malformações congênitas associadas ao diabetes. Clin Obstet Ginecol Am Norte. 1996; 1:31-49.

6. Olofsson P, Liedholm H, Sartor G, Sjoberg NO, Svenningsen $\mathrm{N}$, Ursing $\mathrm{D}$. Diabetes and pregnancy. A 21 year swedish material. Acta Obstet Gynecol Scand (Suppl) 1984; 122:3-62.

7. Daponte A, Guidozzi F, Moisuc D, Marineanu A. Management of diabetic pregnant patients in a tertiary center in the developing world. Int $\mathrm{J}$ Gynaecol Obstet 1999; 64:141-6.

8 Pereira BG, Faúndes A, Parpinelli MA, Pires HB, Cecatti JG. Evolução e controle do diabetes durante a gestação. Rev Ciência Méd. In press.

9. Lubchenco LO, Hansman C, Dressler M, Boyd E. Intrauterine growth as estimated from liveborn birth weight data at 24 to 42 weeks of gestation. Pediatrics 1963; 32:793-6.

10.Pereira BG, Faúndes A. Evolução e controle do diabete melito durante a gestação. Rev Ginecol Obstet 1995; 1:29-35.

11. Oliveira AMB, Camano L, Delascio D. Diabetes e Gravidez. 1ª ed. São Paulo: Sarvier, 1988; p.104.

12.Von Kries R, Kimmerle R, Schmidt JE, Hachmeister A, Bohm O, Wolf HG. Pregnancy outcomes in mothers with pregestational diabetes: a populationbased study in North Rhine (Germany) from 1988 to 1993. Eur J Pediatr 1997; 156:963-7.

13.Pedersen JF, Molsted-Pedersen L. Early growth retardation in diabetic pregnancy. Br Med J 1979; $1: 18-9$.

14.Reece EA, Leguizamon G, Honko C. Pregnancy performance and outcomes associated with diabetic nephropathy. Am J Perinatol 1998; 15:413-21.

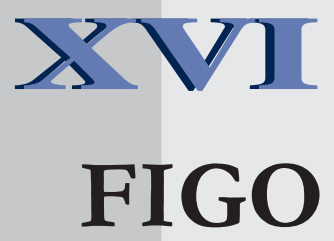

World Congress of Ginecology and Obstetrics

Washington D.C.

september $3-8,2000$

Tel: 514/286-0855 - Fax: 514/286-6066 